\title{
Exploring the Potential of Endoscopic Examinations Using the Kyoto Scoring System for Risk Evaluation of Gastric Carcinoma in Patients with Gastritis
}

\author{
Yasuhiro Nakmaura* and Uch Somary
}

Sun International Clinic, Phnom Penh, Cambodia

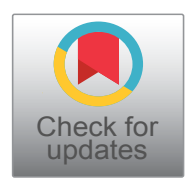

*Corresponding author: Yasuhiro Nakmaura, MD, MPH, Sun International Clinic, House No. 18, Street 302, Sangkat Beoung Keng Kong 1, Khan Chamkamon, Phnom Penh, Cambodia 12000, Cambodia, E-mail: yasuhiro.n1112@gmail.com

\begin{abstract}
Background: Cambodia is considered to be endemic to chronic gastritis due to Helicobacter pylori (HP). Prevalence of HP is more than $70 \%$ inferred from the literature of neighboring countries. However, Cambodia, being a developing nation, doesn't have enough resources for the screening of HP. On the other hand, after the Kyoto Global Consensus in 2015 , risk evaluation of gastric carcinoma based on endoscopic findings with image-enhanced modalities and magnification allows diagnosis of gastritis with a high degree of accuracy. Also, the Kyoto scoring system using five endoscopic features (atrophy, diffuse redness, intestinal metaplasia, enlarged folds, and nodularity) has been proposed as a simplified method.

Method: We retrospectively reviewed the images of 62 patients with upper Gastro Endoscopic (GI) examinations from April 2014 to August 2016 at Sun International Clinic, Cambodia. The Kyoto scoring system and the Operative Link on Gastritis Assessment (OLGA) staging system are evaluated to see the correlation between them using SAS program and determine a cut-off point of the Kyoto scoring system.
\end{abstract}

Result: Mean age (46.2) [minimum: 23, maximum: 72], male/ female (33/29). The endoscopic findings are as follows; The OLGA staging system (Stage 0: 11, Stage I: 14 , Stage II: 26 , Stage III: 8, Stage IV: 3), evaluated determinants of the Kyoto score (atrophy: 41 , enlarged/tortuous fold: 8 , nodularity: 0 , intestinal metaplasia: diffuse redness: 21. High-risk (Stage III \& IV)/low-risk (Stage I \& II) group based on the OLGA staging system: 11/51. Spearman's Rank Order Correlation between them is 0.737 , suggesting a strong correlation. The suggested cut-off point in the Kyoto scoring system is $6(p \leq 0.001)$.

Conclusion: There is a high potential for endoscopic screening using the Kyoto scoring system for risk evaluation of gastric carcinoma. The Kyoto scoring system can make screening less cumbersome and less expensive, and be employed in developing countries with constrained resources.

\section{Keywords}

Helicobacter pylori, Gastritis, Gastric cancer, Kyoto scoring system, OLGA staging system

\section{Introduction}

Helicobacter pylori (HP) is a gram-negative bacterium discovered by Barry Marshall and Robin Warren in 2005 [1]. It is well known that HP infection contributes to cause chronic gastritis, peptic ulcer, Mucosa-Associated Lymphoid Tissue (MALT) lymphoma, gastric cancer and idiopathic thrombocytopenia [2]. Also, atrophic gastritis and intestinal metaplasia are risk factors for gastric ulcers and gastric cancer [3]. Therefore, an accurate risk classification system using these findings for HP related gastric cancer is meaningful to treat and diagnose.

The global prevalence of HP infection is more than $50 \%$ [4]. In developing countries especially in Asia, HP infection is markedly more prevalent [5]. Cambodia, being a developing nation, is considered to be endemic to chronic gastritis due to HP. Although data about prevalence of HP and gastritis in Cambodia does not exist, it is estimated that prevalence of HP is more than $70 \%$ inferred from the literature of neighboring countries [6]. The high prevalence of HP infection suggests that public-health interventions should be considered. Also, Cambodia doesn't have enough resources for the screening of HP including skilled endoscopist and latest equipment. These situations lead to persistent high morbidity of HP in this country.

To diagnose and stratify patients based on ones' risk of gastric cancer, histological staging systems such as the

Citation: Nakmaura Y, Somary U (2017) Exploring the Potential of Endoscopic Examinations Using the Kyoto Scoring System for Risk Evaluation of Gastric Carcinoma in Patients with Gastritis. Int J Oncol Res 1:001.

Received: July 25, 2017; Accepted: October 30, 2017; Published: November 01, 2017

Copyright: (c) 2017 Nakmaura Y, et al. This is an open-access article distributed under the terms of the Creative Commons Attribution License, which permits unrestricted use, distribution, and reproduction in any medium, provided the original author and source are credited. 
Operative Link on Gastritis Assessment (OLGA) system [7] and the Operative Link on Intestinal Metaplasia (OLGIM) [8] are useful for risk stratification. These staging systems provide relevant clinical information. The stage of gastritis results from the combination of the extent of atrophy (scored histologically) with its topographical location (resulting from the mapping protocol).

On the other hand, technology in endoscopy has been developed dramatically. Recently, using magnified endoscopy, clinician can obtain diagnosis from its image as accurate as pathological diagnosis. In 2015, The Kyoto Global Consensus Meeting was convened to develop global consensus on classification of chronic gastritis and duodenitis, appropriate diagnostic assessment of gastritis and recommended treatment HP gastritis [9]. In addition, the Kyoto scoring system of gastritis based on endoscopic findings has been proposed [10]. However, there are little evidences to address the efficacy of classification by the Kyoto scoring system.

Since the Kyoto scoring system can make screening less cumbersome and less expensive, if the Kyoto scoring system would provide equivalent information with pathological findings for risk evaluation of gastric carcinoma, the Kyoto scoring system has high potential for endoscopic screening in developing countries with constrained resources.

Table 1: The OLGA staging system. Stage is scored in following way: 1) Scoring atrophy at each single antral/angular biopsy level (row) and corpus biopsy level (column); 2) Calculating mean of degree of atrophy in antral/angular level (A) and corpus level (C); 3) The final prevalence of atrophy at each compartment is calculated and scored on a four-tiered scale (no atrophy $=0 \%$ : score $=0$, mild atrophy $=1-30 \%$ : score $=$ 1 , moderate atrophy $=31-60 \%$ : score $=2$, severe atrophy $=>$ $60 \%$ : score $=3$ ) [7].

\begin{tabular}{|l|l|l|l|l|}
\hline & C0 & C1 & C2 & C3 \\
\hline A0 & Stage 0 & Stage I & Stage II & Stage III \\
\hline A1 & Stage I & Stage I & Stage II & Stage III \\
\hline A2 & Stage II & Stage II & Stage II & Stage IV \\
\hline A3 & Stage III & Stage III & Stage IV & Stage IV \\
\hline
\end{tabular}

\section{Method}

We retrospectively investigated 62 cases of chronic gastritis at Sun International Clinic, Phnom Penh, Cambodia, with upper Gastroendoscopic (GI) examinations from April 2014 to August 2016 using upper Gl endoscope (GIF-H170, Olympus, Tokyo). Five key features from the Kyoto scoring system and the OLGA staging system were evaluated to see the correlation between them using SAS program and determine a cut-off point of the Kyoto scoring system. All patients were scored according to the Kyoto scoring system by one non-expert endoscopists. HP status was not evaluated.

The OLGA staging system is based on pathological findings [7]. Different biopsy locations have been suggested for both the oxyntic and the antral mucosa have to be examined. Therefore, taking 5 biopsy samples ( 3 from them ucosecreting compartment and 2 from the oxyntic compartment) are recommended [7]. Table 1 shows the way to classify into the OLGA stages. For each biopsy sample, each strip is labeled according to its site of origin (antral/ angular $=\mathrm{A}$, corpus $=\mathrm{C}$ ). Assessing atrophy at each compartment level by calculating each mean value, the final prevalence of atrophy at each compartment is calculated and scored on a four-tiered scale (no atrophy $=0 \%$ : score $=0$, mild atrophy $=1-30 \%$ : score $=1$, moderate atrophy = 31-60\%: score $=2$, severe atrophy $=>60 \%$ : score $=3$ ) [7] high-risk (III/IV) The OLGA stages is consistently associated Invasive or intraepithelial gastric neoplasia [7].

The Kyoto scoring system evaluates atrophy, enlarged fold, nodularity, intestinal metaplasia and diffuse redness, and calculates risk score of gastric carcinoma [Range: 0-8] [9] (Figure 1). Atrophy is evaluated based on the degree of atrophy by Kimura-Take moto classification range C-O, C-I, C-II, C-III, O-I, O-II, and O-III. C-0 and $\mathrm{C}-1$ score $1, \mathrm{C}-2$ and $\mathrm{C}-3$ score 2 and $\mathrm{O}-1, \mathrm{O}-\mathrm{II}$ and $\mathrm{O}$-III score 3. Intestinal metaplasia is evaluated based on its distribution. [Score 0 (none), score 1 (antrum), score 2 (antrum-body)] Enlarged fold is evaluated by its

\section{$\underline{\text { Kyoto scoring system }}$}

- Atrophy: 0 (C0-1), 1 (C2-3), 2 (O1-OP)

- Intestinal Metaplasia: 0 (none), 1 (antrum), 2 (antrum-body)

- Enlarged Fold: $0(<4 \mathrm{~mm}), 1(>5 \mathrm{~mm})$

- Nodular: 0 (none), $1(+)$

- Diffuse Redness: 0 (none), 1 (mild), 2 (severe)

Figure 1: The Kyoto scoring system. Atrophy is evaluated based on the degree of atrophy by Kimura-Take moto classification range C-0, C-I, C-II, C-III, O-I, O-II, and O-III. C-0 and C-1 score 1, C-2 and C-3 score 2 and O-1, O-II and O-III score 3. Intestinal Metaplasia is evaluated based on its distribution. [Score 0 (none), score 1 (antrum), score 2 (antrum-body)] Enlarged Fold is evaluated by its thickness. [Score $0(<4 \mathrm{~mm})$, score $1(>5 \mathrm{~mm})$ ] Nodular scores 1 if it exists and 0 if not. Diffuse Redness is evaluated by the degree of visibility of Regular Arrangement of Collecting Venules (RAC) at body. [RAC (+): score 0, RAC (partly): score1, RAC (-): score 2] [9]. 
thickness. [Score $0(<4 \mathrm{~mm})$, score $1(>5 \mathrm{~mm})$ ] Nodular scores 1 if it exists and 0 if not. Diffuse redness is evaluated by the degree of visibility of Regular Arrangement of Collecting Venules (RAC) at body. [RAC (+): score 0 , RAC (partly): score1, RAC (-): score 2] [10]. Although the Kyoto scoring system has been developing as a new diagnostic scale for gastritis, no cut-off value is reported for risk evaluation for gastric cancer.

\section{Result}

The mean age in this sample was 46.2 years old [minimum: 23, maximum: 72 ]. The proportion of males $(n=$ 33) was higher than females $(n=29)$. Table 2 shows the OLGA risk stratification. The number of high-risk group for gastric cancer is 11 as well as that of low-risk group is 51. Using the Kyoto scoring system, 5 factors were evaluated as follows; Atrophy ( $n=41)$, Enlarged fold ( $n=$ $8)$, Nodularity $(n=0)$, Intestinal metaplasia $(n=35)$, Diffuse redness $(n=21)$. Then, we computed scores (Table $3)$. The concordance of the OLGA and the Kyoto scoring system was evaluated by Spearman's Rank order correlation, which was $0.738(p=0.01)$. If we set cut-off value for the Kyoto scoring system as 6 , high or low-risk groups by the Kyoto scoring system was compatible with those by the OLGA scoring system. [Sensitivity: $100.00 \%$ ( $95 \% \mathrm{Cl}-71.51 \%$ to $100.00 \%$ ), specificity: $100.00 \%$ (95\% $\mathrm{Cl}-93.02 \%$ to $100.00 \%)]$. The distributions using the Kyoto scoring system and the OLGA are shown in (Figure 2), which revealed significant correlation between the Kyoto scoring system and the OLGA $(p=0.01)$.

Table 2: The OLGA stage in this study $(n=62)$.

\begin{tabular}{|l|l|}
\hline OLGA stage & Frequency $(\mathbf{n}=\mathbf{6 2})$ \\
\hline Stage 0 & $11(17.7 \%)$ \\
\hline Stage 1 & $14(26.6 \%)$ \\
\hline Stage 2 & $26(41.9 \%)$ \\
\hline Stage 3 & $8(12.9 \%)$ \\
\hline Stage 4 & $3(4.8 \%)$ \\
\hline
\end{tabular}

\section{Olga Score}

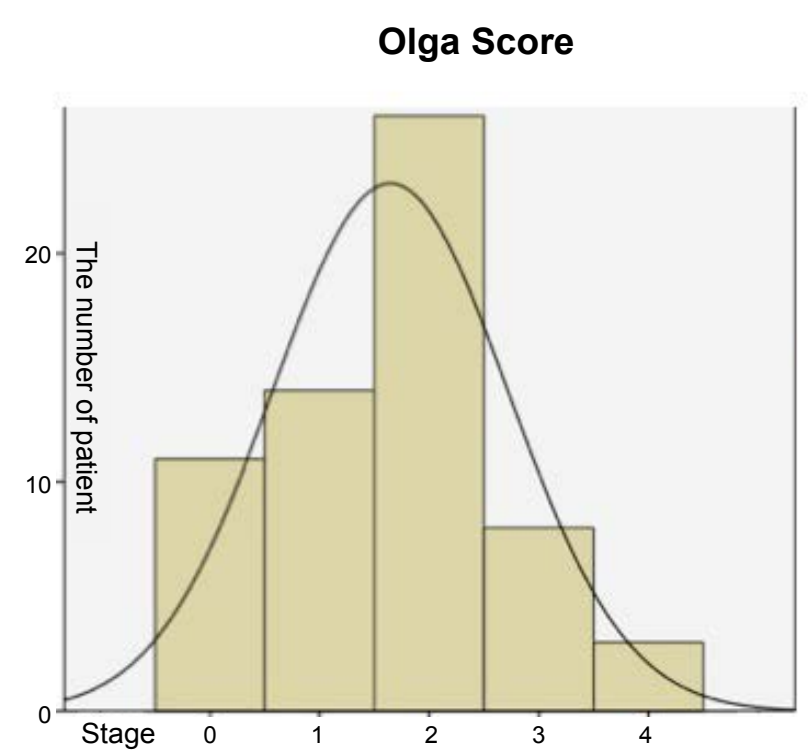

\section{Discussion}

In this study, $82.3 \%$ of patients were estimated as low risk of gastric carcinoma for the OLGA. Before we analyzed these data, we hypothesized that we could apply this percentage and estimate cut-off value on the Kyoto scoring system if these scoring/staging systems were compatible. As the result, we identified compatibility between the OLGA and the Kyoto scoring system, and estimated cut-off value as 6 for the Kyoto scoring system. Figure 2 showed that each distribution was apparently different. However, using cut-off value " 6 " for the Kyoto scoring system, we found significant correlation between the Kyoto scoring system and the OLGA.

The identification and management of chronic gastritis are important for preventing from developing gastric cancer. The degree of atrophy with intestinal metaplasia contributes to current and future risk for developing differentiated gastric cancer $[3,11]$. The cancer rate based on Kimura-Take moto classification is $0 \%$ for C-I type, $0.25 \%$ for C-II, $0.71 \%$ for C-III, $1.32 \%$ for O-I, 3.70\% for O-II, and $5.33 \%$ for O-III, which showing a significant increase in the detection rate as atrophy progresses [12]. The Kyoto scoring system attempts to classify a risk of gastric cancer with five endoscopic findings associated with HP infection [10]. Severity and extent of atrophic

Table 3: The Kyoto scoring system in this study $(n=62)$.

\begin{tabular}{|l|l|}
\hline Kyoto score & Frequency $(\mathbf{n}=\mathbf{6 2})$ \\
\hline 0 & $16(25.8 \%)$ \\
\hline 1 & $10(16.1 \%)$ \\
\hline 2 & $5(8.1 \%)$ \\
\hline 3 & $5(8.1 \%)$ \\
\hline 4 & $9(14.5 \%)$ \\
\hline 5 & $6(9.7 \%)$ \\
\hline 6 & $6(9.7 \%)$ \\
\hline 7 & $5(8.1 \%)$ \\
\hline 8 & $0(0 \%)$ \\
\hline
\end{tabular}

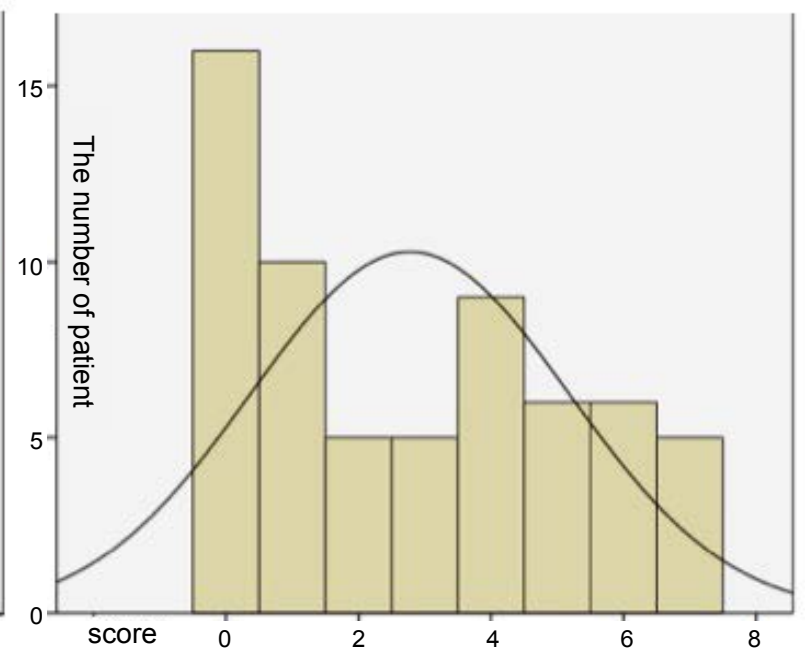

Figure 2: Distribution of patient in this study using the OLGA staging system and the Kyoto scoring system. 
gastritis and intestinal metaplasia are well established as indicators of the increased risk for developing gastric cancer $[9,13]$. Distinctions between the OLGA and the Kyoto scoring system are that the OLGA uses only the atrophy score alone and the Kyoto scoring system is based on endoscopic findings instead of pathological diagnosis like the OLGA. This study tried to confirm the degree of correlation between these two staging system so that we could determine who was high/low-risk for gastric cancer.

After diagnosing HP positive gastritis, eradication of HP is strongly recommended to decrease the gastric cancer risk $[3,14]$. In a meta-analysis of randomized controlled trials using HP positive healthy asymptomatic individuals, the relative risk for developing gastric cancer with eradication therapy compared with placebo or no treatment was 0.66 [15]. After eradication therapy, the total score of the Kyoto scoring system would be expected to decrease due to improve diffuse redness and hypertrophy of gastric folds. However, some problems in developing countries are to be burdens to patients living in those countries. First of all, there is insufficient insurance system. Even if some patients without insurance were diagnosed as HP positive gastritis and should have taken eradication therapies, they could not afford on their own and were not willing to take medication. Secondly, skills and equipment concerning endoscopy are insufficient. It is difficult to find skilled endoscopist. Also, endscopist mostly uses conventional endoscopy and magnified endoscopy is not available. Therefore, addressing accurate risk in simple way is important for patients to be motivated to take further treatment and examination and for clinicians to diagnose correctly. This study sheds light on the potential of the Kyoto scoring system for endoscopic screening in developing countries.

Using cut-off value can be a potential method to screen a larger population in an efficient and inexpensive way, which is vital for developing nations. In this study, endoscopic findings were evaluated not by well-experienced endoscopist (Experience of endoscopic procedure is 5001000 cases) because endoscopists in Cambodia were not skilled. Although committee of the Japanese Society of Gastroenterology recommend for endoscopists to examine patient with magnified endoscopy to use theKyoto scoring system and conclude cconventional endoscopy is mostly an inadequate tool for diagnosing atrophy and intestinal metaplasia [9], this results revealed a conventional endoscopy was useful for risk-classification of gastric cancer to obtain equivalent results of pathological findings if we used cut-off value of theKyoto scoring system. However, at this point, it remains mandatory that a biopsy should be carried out in addition to endoscopic diagnosis. More studies should be conducted in future with larger sample size so that this staging system provides relevant clinical information.

\section{Limitations}

This study contains small number of cases. The Kyo- to scoring system categorizes the endoscopic characteristics of HP infection-associated gastritis and identifies patterns associated with a high risk of gastric cancer [16]. We could not obtain HP status data for all patients because of its cost. Also, some patients are not willing to take eradication therapy due to its cost and their knowledge that falls short of understanding importance of eradication therapy. Therefore, future studies will need to compare the gastritis scores before and after eradication as a prospective study.

\section{Conclusion}

In conclusion, we addressed that the scores of the Kyoto scoring system provided clinically useful to stratify patients with high risk for early-stage gastric cancer. This result gives light on the potential of conventional endoscopy performed by non-expert endoscopist, and enable clinicians in developing countries easily to categorize risk of gastric carcinoma. Further investigations are needed to confirm correspondence rate in larger sample.

\section{Conflict of Interest}

I have no personal or financial interests to declare. I have no financial support from an industry source at the current study.

\section{Financial Arrangements}

Employment.

\section{References}

1. Ahmed N (2005) 23 years of the discovery of Helicobacter pylori: Is the debate over? Ann Clin Microbiol Antimicrob 4: 17.

2. Peter Malfertheiner, Francis Megraud, Colm A O'Morain, John Atherton, Anthony TR Axon, et al. (2012) Management of Helicobacter pylori infection-the Maastricht IV/ Florence Consensus Report. Gut 61: 646-664.

3. Uemura N, Okamoto S, Yamamoto S, Matsumura N, Yamaguchi S, et al. (2001) Helicobacter pylori infection and the development of gastric cancer. N Engl J Med 345: 784-789.

4. Graham DY (2014) History of Helicobacter pylori, duodenal ulcer, gastric ulcer and gastric cancer. World J Gastroenterol 20: 5191-5204.

5. Hunt RH, Xiao SD, Megraud F, Leon-Barua R, Bazzoli F, et al. (2011) Helicobacter pylori in developing countries. World gastroenterology organisation global guideline. J Gastrointestin Liver Dis 20: 299-304.

6. Fock KM, Ang TL (2010) Epidemiology of Helicobacter pylori infection and gastric cancer in Asia. J Gastroenterol Hepatol 25: 479-486.

7. Rugge M, Meggio A, Pennelli G, Piscioli F, Giacomelli L, et al. (2007) Gastritis staging in clinical practice: The OLGA staging system. Gut 56: 631-636.

8. Slama SB, Ghachem DB, Dhaoui A, Jomni MT, Dougui MH, et al. (2016) Gastrites chroniques à hélicobacter pylori: Évaluation des systèmes OLGA et OLGIM. Pan Afr Med J 23: 28.

9. Sugano K, Tack J, Kuipers EJ, Graham DY, El-Omar EM, et al. (2015) The Kyoto global consensus report on Helicobacter pylori gastritis. Gut 64: 1353-1367. 
10. Sugimoto $M$, Ban $H$, Ichikawa $H$, Sahara $S$, Otsuka $T$, et al. (2017) Efficacy of the Kyoto Classification of Gastritis in Identifying Patients at High Risk for Gastric Cancer. Intern Med 56: 579-586.

11. Take S, Mizuno M, Ishiki K, Nagahara $Y$, Yoshida $T$, et al (2005) The effect of eradicating Helicobacter pylori on the development of gastric cancer in patients with peptic ulcer disease. Am J Gastroenterol 100: 1037-1042.

12. Hironori Masuyama, Naoto Yoshitake, Takako Sasai, Tetsuya Nakamura, Atsushi Masuyama, et al. (2015) Relationship between the degree of endoscopic atrophy of the gastric mucosa and carcinogenic risk. Digestion 91: 30-36.

13. Ford AC, Forman D, Hunt RH, Yuan Y, Moayyedi P (2014) Helicobacter pylori eradication therapy to prevent gastric cancer in healthy asymptomatic infected individuals: Systematic review and meta-analysis of randomised controlled trials. BMJ 348: 3174.

14. Graham DY, Shiotani A (2005) The time to eradicate gastric cancer is now. Gut 54: 735-738.

15. G M Naylor, T Gotoda, M Dixon, T Shimoda, L Gatta, et al. (2006) Why does Japan have a high incidence of gastric cancer? Comparison of gastritis between UK and Japanese patients. Gut 55: 1545-1552.

16. Khoshnood A, Hakimi $P$, Salman-Roghani $H$, Reza Mirjalili M (2014) Replacement of clarithromycin with azithromycin in triple therapy regimens for the eradication of Helicobacter pylori: A randomized clinical trial. J Med Life 7: 254-259. 